\title{
Investigation of controlled ultrashort pulse dye laser for femtosecond nonlinear spectroscopy
}

\author{
Y. Ishida and T. Yajima \\ Institute for Solid State Physics, University of Tokyo, Roppongi, Minato-ku, Tokyo 106, Japan
}

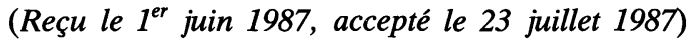

\begin{abstract}
Résumé. - Les caractéristiques d'un système simple et pratique à impulsions laser pour la spectroscopie non linéaire transitoire vers 100 fs et en dessous sont décrites. Le système est basé sur un laser à colorant continu, à blocage en phase des modes hybride, avec une cavité à extraction d'impulsions, à haute stabilité, à grande accordabilité et à propriétés de phase spécifiques, produisant aussi bien des impulsions nettes limitées par transformée de Fourier que des impulsions en " couic » (à fréquence instantanée variable, «chirped »). Le système laser est utilisé pour démontrer la faisabilité d'employer des impulsions en "couic » à la mesure à haute résolution temporelle des durées de relaxation en milieu condensé par mélange transitoire à quatre ondes dégénérées.
\end{abstract}

\begin{abstract}
Characteristics of a simple but practical ultrashort pulse laser system for transient nonlinear spectroscopy around and below $100 \mathrm{fs}$ are described. The system is based on a hybridly mode-locked, cavitydumped cw dye laser, with high stability, broad tunability and specific phase properties, producing both clean transform-limited and chirped pulses. The laser system is applied to demonstrate the feasibility of using chirped pulses in high time-resolution measurement of relaxation times in condensed matter by means of degenerate transient four-wave mixing.
\end{abstract}

\section{Introduction.}

Development of high-performance ultrashort pulse lasers is of importance for their application to ultrafast spectroscopy in the time-domain, where a variety of dynamical processes in materials are studied [1]. For this purpose, it is required to generate well-controlled ultrashort pulses, not only in shortening the pulse width but also with regard to stability, tunability and, in addition, pulse shape and phase properties.

Generation of clean linearly-chirped pulses is important in two respects. One is to generate clean transform-limited pulses by compression technique. The other is to apply the pulses to a new method of transient nonlinear spectroscopy, where a time-resolution much shorter than the pulse width is obtainable [2, 3]. For the experimental study of basic features of this method in comparison with the conventional one, it is desirable to have a reliable laser source capable of producing both clean transform-limited pulses and clean linearly-chirped pulses.

Even at the highly-developed stage of present laser technique, there are only a few laser systems that satisfy the above requirements. The colliding pulse mode-locked (CPM) ring dye laser has generated stable, transform-limited pulses of $27 \mathrm{fs}$ by introducing a prism sequence inside the cavity [4]. However, the pulse properties become complicated, as the saturable absorber dye degrades in a relatively short time. Further, tuning range is limited by the absorption band of the absorber dye. An alternative approach is to use the hybridly mode-locked, cavitydumped, cw dye laser coupled with a single-stage fiber compressor which produces tunable femtosecond pulses [5]. The advantages of this system are not only its frequency tunability, but also ease of controlling the pulse width and phase property. This system can readily be realized by modifying a commercial synchronously-mode-locked dye laser. These characteristics are not strongly affected by the degradation of the absorber dye compared to that of the CPM ring dye laser.

In this paper, we present first the characteristics of our laser system based on the latter scheme described above, which has been developed for the study of transient nonlinear spectroscopy. It will be shown 
that, by careful control of operating conditions, the dye laser produces clean Gaussian-like pulses over a broad tuning range, which is important for efficient fiber pulse compression. By coupling with a singlestage fiber compressor, both tunable transform-limited and linearly-chirped pulses can be obtained from the same source.

Under certain operating conditions, positive linear-chirp is observed in the pulses directly from the laser oscillator over the oscillating frequency range. The mechanism will be discussed in terms of self-phase modulation (SPM) inside the cavity.

This laser system is also applied to demonstrate experimentally the feasibility of using chirped pulses in high time-resolution transient spectroscopy. The degenerate four-wave mixing scheme in a dye solution and in a semiconductor material was used in the experiment for relaxation studies. The results and their interpretation will be given.

\section{Experimental procedure.}

The experimental setup is shown in figure 1. The laser used for this work is based on a hybridly (passively and synchronously) mode-locked, cavitydumped, rhodamine $6 \mathrm{G}$ dye laser which can operate at repetition rates from $4 \mathrm{MHz}$ down to single-shot. The pumping source is a cw mode-locked $\mathrm{Ar}$ ion laser at a repetition rate of $82 \mathrm{MHz}$.

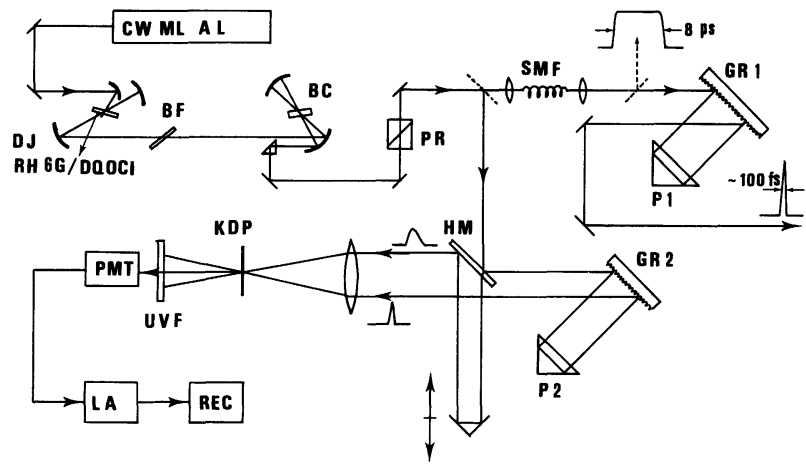

Fig. 1. - Experimental arrangement for pulse compression and SHG cross-correlation measurement. AL : argon ion laser ; BC : Bragg cell ; BF : birefringence filter ; DJ : dye jet ; LA : lock-in amp. ; PR : polarization rotator ; SMF : single mode fiber.

The cavity consists of two sets of folded focusing mirrors, with radii of curvature of $5 \mathrm{~cm}$ for the laser dye jet $(0.2 \mathrm{~mm}$ thick $)$ and of $10 \mathrm{~cm}$ for the cavity dumper (Bragg cell, $3 \mathrm{~mm}$ thick).

A saturable absorber dye (DQOCI) for passive mode-locking was mixed in the laser dye solution $\left(2 \times 10^{-3} \mathrm{M}\right.$ in ethylene glycol). The absorber concentration was kept within $2 \times 10^{-5} \mathrm{M}$. With the DQOCI concentration of $1.5 \times 10^{-5} \mathrm{M}$, the time- averaged threshold pumping power, $P_{\text {th }}$, for oscillation was $350-380 \mathrm{mw}$, while with synchronous modelocking alone, it was about $100 \mathrm{mw}$. The pulses are continuously tunable from $575-645 \mathrm{~nm}$ by using a single-plate birefringence filter $0.3 \mathrm{~mm}$ thick. Experiments were carried out under the condition of relative pumping power of $P / P_{\text {th }}=1.1-2.0$.

The pulse compression system consisting of an optical fiber and a dispersive delay line is similar to that reported previously [5]. Linearly polarized input pulses traversed a polarization-preserving single-mode optical fiber with a length of $2.5 \mathrm{~m}$ and a core diameter of $3.6 \mu \mathrm{m}$. With a $20 \mathrm{X}$ microscope objective lens, the coupling efficiency of input pulses was over $60 \%$. The output pulses recollimated with a $20 \mathrm{X}$ microscope objective lens were passed through a grating of 1200 lines $/ \mathrm{mm}$ and a right-angle corner-reflector. The optimum length for minimum compressed pulse width is adjusted by changing the separation between the grating and the corner-reflector. The total transmittance of the compressor system was $\sim 20 \%$. The behaviour of the chirped pulses was also measured using another dispersive delay line as in the pulse compressor system.

The pulse width was measured with a conventional non-collinear SHG autocorrelator with a KDP crystal $0.1 \mathrm{~mm}$ thick. The SHG cross-correlation technique was also used to measure the asymmetry of the laser pulse. These measurement systems can operate both at a slow scan rate for obtaining the precise correlation trace and at a rapid scan rate $(20 \mathrm{~Hz})$ for continuously monitoring the correlation trace.

\section{Results and discussion.}

3.1 Generation OF CONTROLled PUlSES AND PULSE COMPRESSION. - The basic features of our system have already been reported [4], but we reproduce its main results briefly in 3.1 for a basis.

Without the saturable absorber, the laser system shown in figure 1 can operate in the conventional synchronous mode-locking. The average output power of $70-80 \mathrm{mw}$ at the repetition rate of $4 \mathrm{MHz}$ was obtained for the pumping power of $0.8 \mathrm{~W}$. The SHG autocorrelation trace always showed a broad wing with a coherence spike at the peak. The typical pulse width was $6 \mathrm{ps}$ ( $\mathrm{sech}^{2}$ shape) around $600 \mathrm{~nm}$. After passing these pulses through the fiber compressor, the compressed pulse width was about 0.5 ps. The correlation trace showed a very broad wing as it did for the input pulse. The measured compression ratio is much smaller than the value by calculation.

On the other hand, the hybrid mode-locking operation largely improved the characteristics of the pulse, i.e., the stability, the pulse shape and its width. We found that there are two different operating regions for stable mode-locking depending on the pumping power. With the DQOCI absorber 


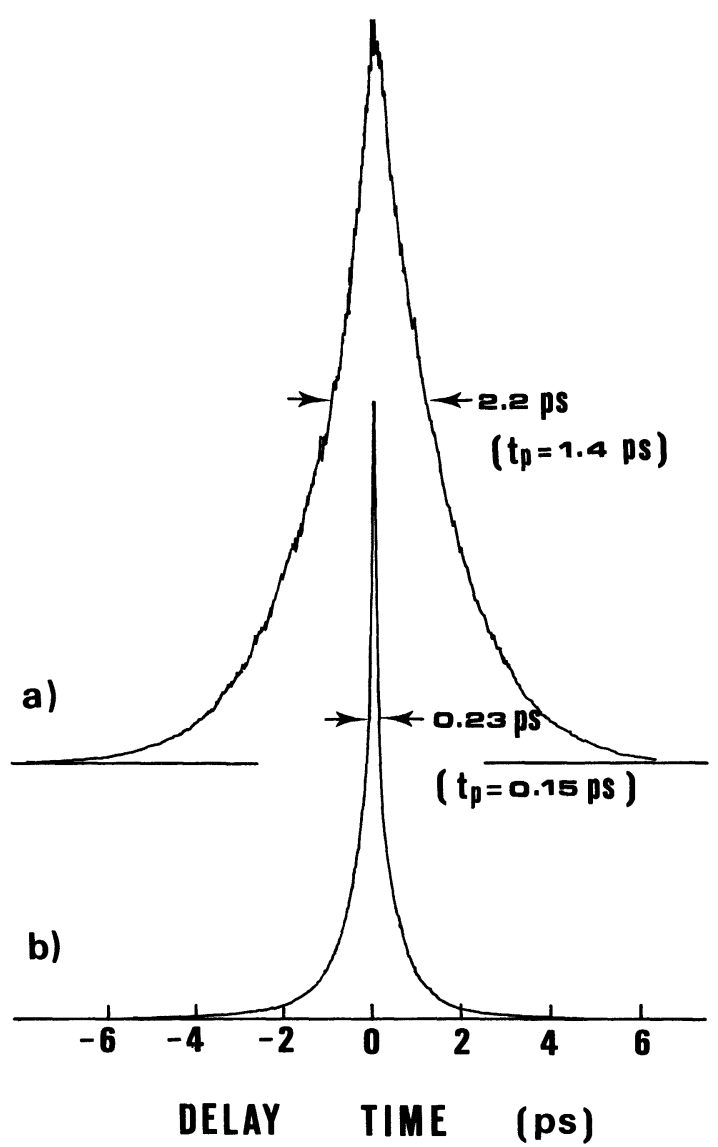

Fig. 2. - SHG autocorrelation traces of input (a) and output (b) pulses to and from the pulse compressor with external optical fiber. The light source is a hybridly modelocked rhodamine $6 \mathrm{G}$ dye laser under usual operation (relative pumping power $P / P_{\text {th }}=2$, DODCI concentration $\left.C_{\mathrm{D}}=6 \times 10^{-6} \mathrm{M}\right)$. The indicated pulse widths $\left(t_{\mathrm{p}}\right)$ are derived by assuming a $\operatorname{sech}^{2}$ shape.

concentration of $6-8 \times 10^{-6} \mathrm{M}$, and the relative pumping power $P / P_{\mathrm{th}}=2$, the pulse width and the average power were 1.4 ps and $30 \mathrm{~mW}$, respectively, as shown in figure $2 a$. Figure $2 b$ shows the SHG autocorrelation trace of the compressed pulses. The pulse width of $0.15 \mathrm{ps}$ is much shorter than that for the synchronous mode-locking alone. This is due to the increase of the effective peak power as a result of the reduction of the energy around the wing.

We further tried to control the pulse shape and its width by adjusting the pumping power level. With $P / P_{\text {th }}=1.1-1.5$, a very clean pulse was obtained as shown in figure $3 \mathrm{a}$. The SHG autocorrelation trace is best fitted by a 1.1 ps (FWHM) Gaussian shape which is plotted with black points. Then, the efficient pulse compression could be realized by using the well-controlled pulses. The compressed pulse of $103 \mathrm{fs}\left(\operatorname{sech}^{2}\right.$ shape) shown in figure $3 \mathrm{~b}$ is very clean and short, even though the input peak power of $3 \mathrm{~kW}$ is smaller than that for the case of figure $2 \mathrm{a}$.

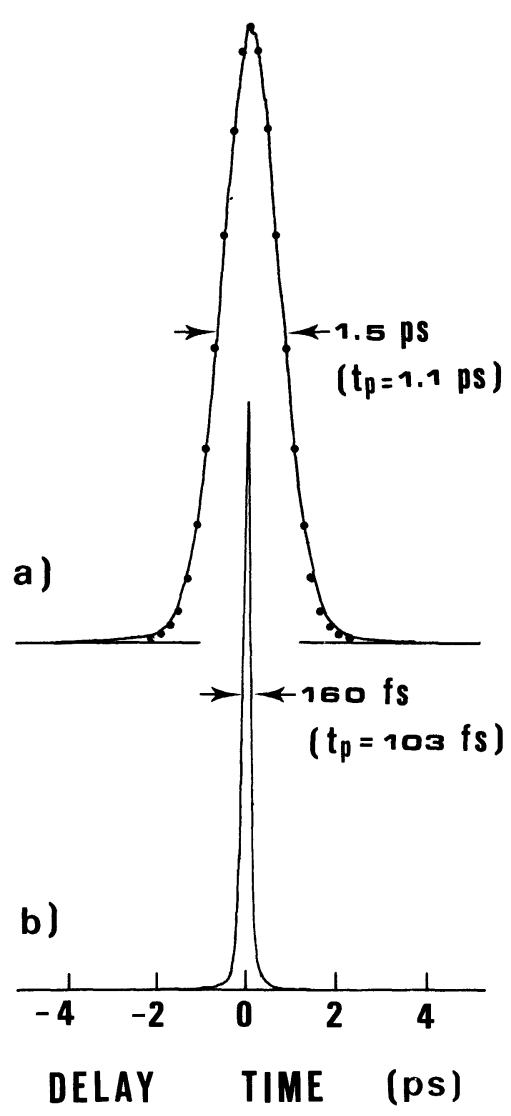

Fig. 3. - SHG autocorrelation traces of input (a) and output (b) pulses to and from the pulse compressor with external optical fiber. The light source is of the same type as in figure 1 but under well-controlled operation $\left(P / P_{\mathrm{th}}=1.5, C_{\mathrm{D}}=6 \times 10^{-6} \mathrm{M}\right)$. The trace (a) is best fitted by a Gaussian pulse shape (black points). In the trace $(\mathrm{b})$, the pulse width $\left(t_{\mathrm{p}}\right)$ is derived by assuming a $\operatorname{sech}^{2}$ shape.

The measured compression ratio is close to the calculated value of 11 . The important point is that, in this laser system, the stable mode-locking region showing the shortest pulse width is broad in pumping power $\left(P / P_{\text {th }}=1.1-1.5\right)$, as in the CPM ring dye laser.

Main reasons for efficient pulse compression are the generation of clean pulse shape with little wings and resulting high peak power. Thus, positive linearchirp ideally occurs over the entire pulse envelope based on the combination of self-phase modulation and the positive group velocity dispersion [6-8]. Experimental evidence for this fact was examined with a measurement of SHG autocorrelation trace of the broadened pulse after passing through the fiber. The measured correlation width of $8 \mathrm{ps}$ showed a triangular shape except the far wings. We can see from this result that the hybridly mode-locked dye laser system is superior to the synchronously modelocked one for fiber pulse compression. 
The shortest pulse width of $90 \mathrm{fs}$ was obtained near $600 \mathrm{~nm}$, under the operating conditions with a peak power of $3.4 \mathrm{~kW}$ and a repetition rate of $0.8 \mathrm{kHz}$. From the measured spectral width, the time-bandwidth product was found to be 0.41 , which is fairly close to but $30 \%$ larger than that of the transform-limited pulse of $\operatorname{sech}^{2}$ shape. By using the spectrum-resolved SHG correlation technique [9, 10], it was also found that the pulses are a little positively chirped. The compressed pulse is frequency tunable in the range of $595-625 \mathrm{~nm}$ as shown in figure 4 . The present dye laser system which can produce tunable, nearly transform-limited and clean chirped pulses is a good candidate for the study of new transient nonlinear spectroscopy as will be discussed in section 4 .

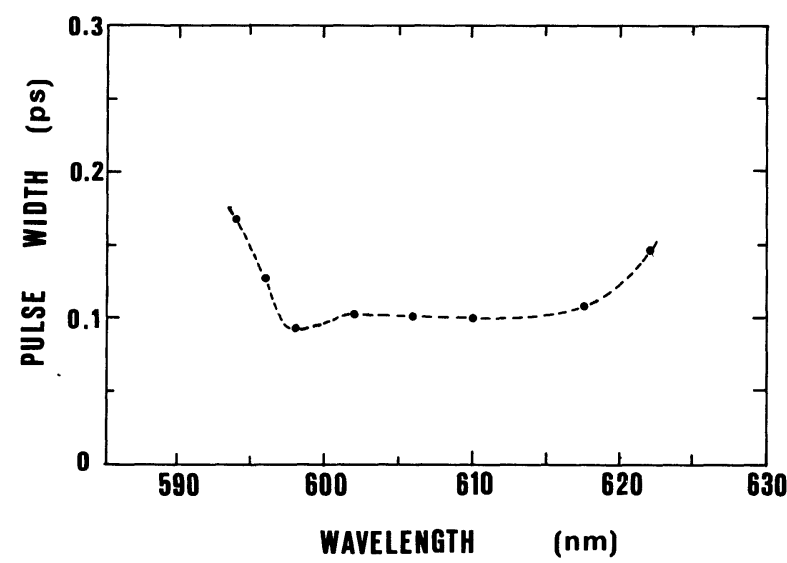

Fig. 4. - Wavelength dependence of the width of the compressed pulse corresponding to figure $3 \mathrm{~b}$.

3.2 OBSERVATION OF POSITIVE LINEAR CHIRP INSIDE THE CAVITY. - It was found that the phase and shape properties of pulses from this laser system depend strongly on the absorber dye concentration as well as the relative pumping power $P / P_{\text {th }}$. New features appeared at higher dye concentration and corresponding higher $P_{\mathrm{th}}$.

With $P / P_{\text {th }}=2$ and a DQOCI concentration of $2 \times 10^{-5} \mathrm{M}$, the average output power was about $15 \mathrm{~mW}$ at the repetition rate of $4 \mathrm{MHz}$. The correlation width of 1.5 ps (FWHM) in figure 5a shows an exponential-like broad wing as in figure $2 \mathrm{a}$. Then, the spectrum exhibited an asymmetric shape (the width of $1.8 \mathrm{~nm}$ ) with a broad wing in the long wavelength side alone as shown in figure $6 \mathrm{a}$. The behaviour is very similar to that obtained previously from the hybridly mode-locked dye laser without the cavity dumper, in which the characteristics of the pulses have been explained by considering slow selfphase modulation (SPM) in the mixed dye solution [9].
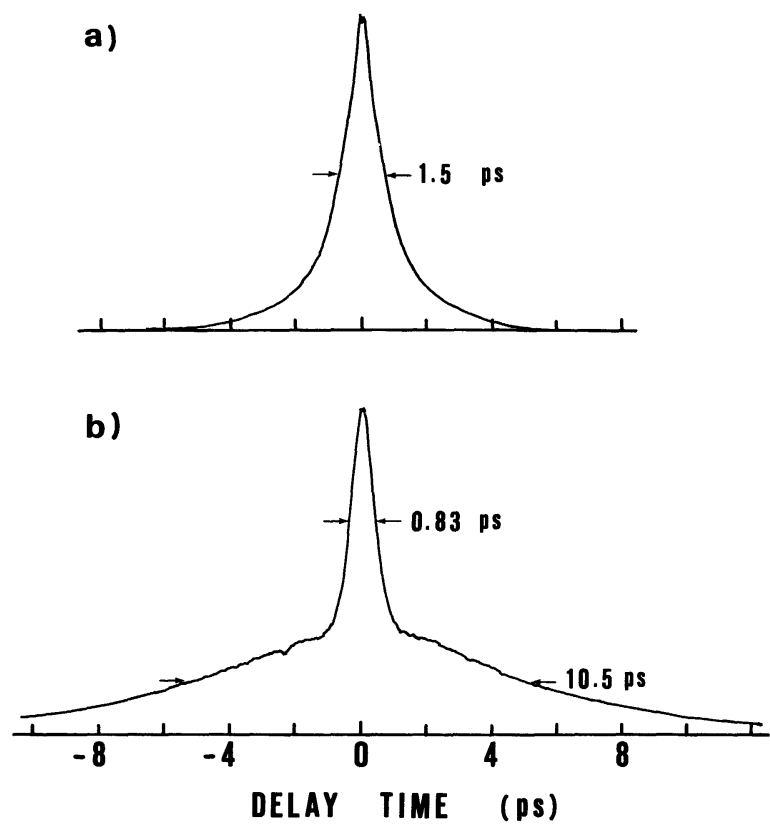

Fig. 5. - SHG autocorrelation traces of input (a) and output (b) pulses to and from the pulse compressor without optical fiber. Laser operating conditions : $P / P_{\text {th }}=2.0$ and $C_{\mathrm{D}}=2 \times 10^{-5} \mathrm{M}$. The indicated widths are the autocorrelation widths.

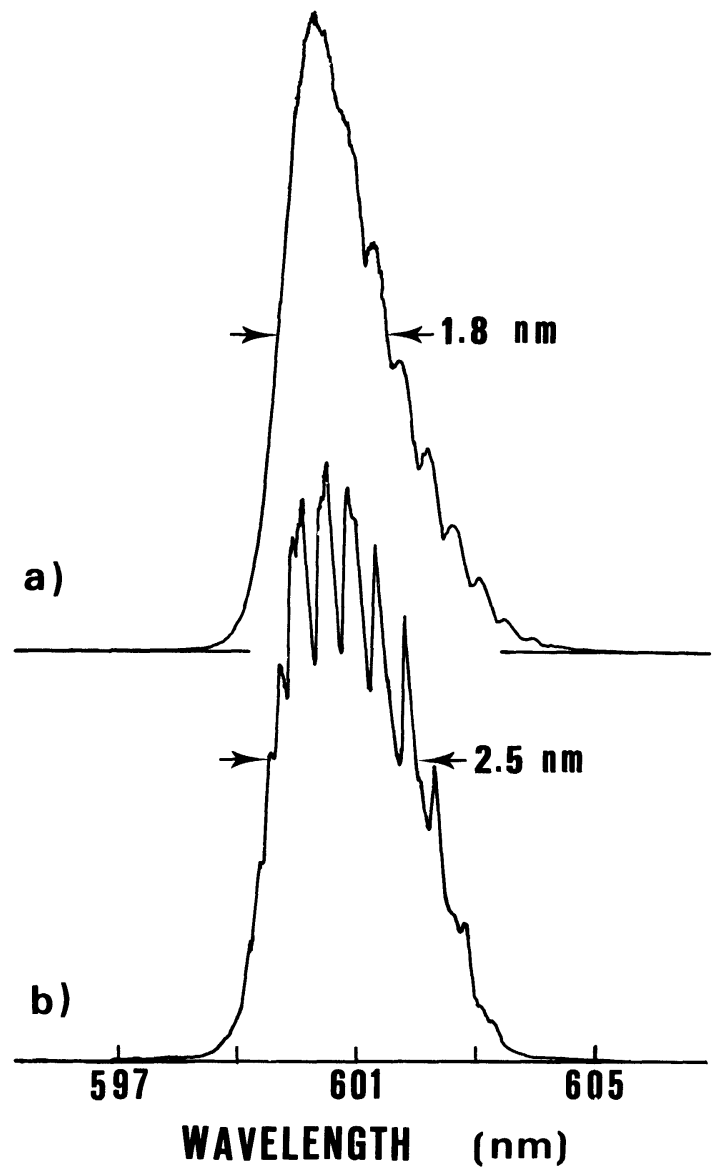

Fig. 6. - Time-integrated spectra of mode-locked pulses. Relative pumping power: (a) $P / P_{\text {th }}=2.0$, (b) $P / P_{\text {th }}=$ 1.5 . 
The SHG autocorrelation trace of the pulse passing through an external grating-prism pair is shown in figure 5b. Except around the peak, the pulse is stretched by the negative dispersive element, which indicates the existence of negative chirp at the wings.

On the other hand, with $P / P_{\text {th }}=1.1-1.5$, stable and clean pulses as in the result of figure $3 a$ are obtained. Figure 7a shows an SHG autocorrelation trace of the pulse before passing through the grating at $P / P_{\text {th }}=1.5$. The pulse width was $1.0 \mathrm{ps}$ $\left(\mathrm{sech}^{2}\right.$ shape) at $600 \mathrm{~nm}$. A weak side-lobe was often observed under the condition of high concentration of the absorber dye $\left(\geq 1 \times 10^{-5} \mathrm{M}\right)$ and high pumping power. The spectrum as shown in figure $6 \mathrm{~b}$ exhibited a nearly symmetric shape with the width of $2.5 \mathrm{~nm}$ (FWHM), which was limited by the bandwidth of the birefringence filter used. The timebandwidth product of 2.1 is 6.7 times larger than that of the transform-limited $\operatorname{sech}^{2}$ pulse shape. This suggests the existence of large chirp. Note that both the spectral shape and the shape of the correlation

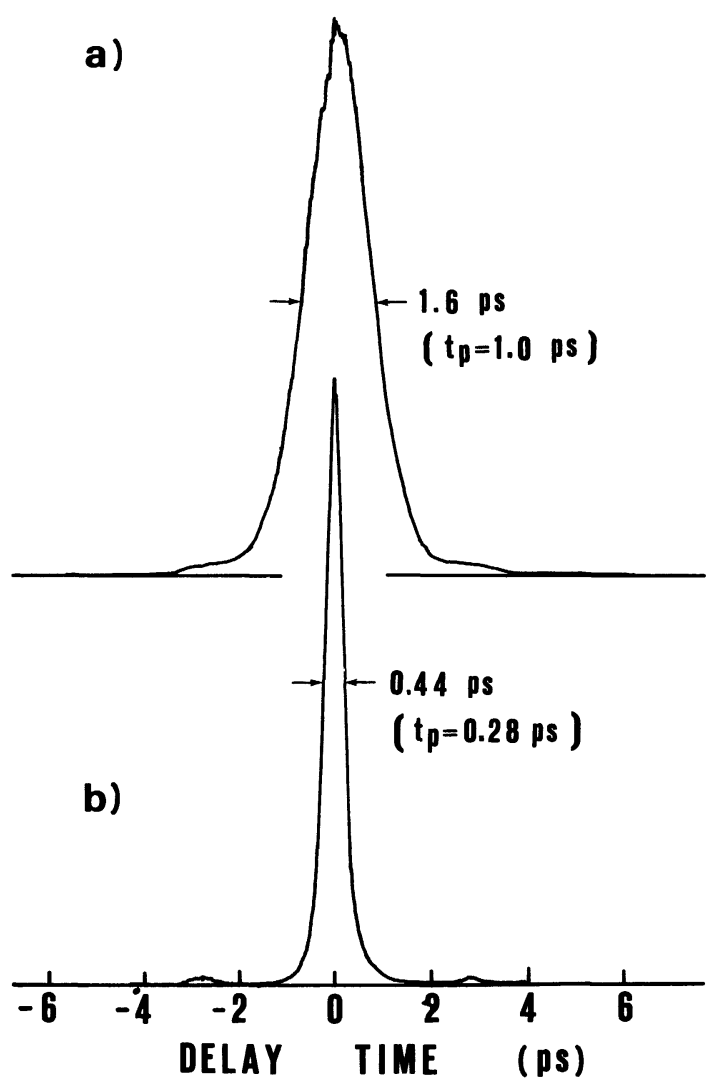

Fig. 7. - SHG autocorrelation traces of input (a) and output (b) pulses to and from the pulse compressor without optical fiber. Laser operating conditions : $P / P_{\text {th }}=1.5$ and $C_{\mathrm{D}}=2 \times 10^{-5} \mathrm{M}$. The indicated pulse widths $\left(t_{\mathrm{p}}\right)$ are derived by assuming a $\operatorname{sech}^{2}$ shape. The results indicate the existence of positive linear chirp in the laser output.

REVUE DE PHYSIQUE APPLIQUÉE. - T. 22, N` 12, DÉCEMBRE 1987 trace drastically change, when $P / P_{\text {th }}$ changes across the level of 1.5-1.6, like a kind of bistable operation. The average output power was $7 \mathrm{~mW}$. Periodic structure on the spectrum envelope in figures $6 \mathrm{a}$ and $6 \mathrm{~b}$ is due to the etalon effect of the birefringence filter $(0.3 \mathrm{~mm}$ thick) used as a tuning element. Figure $7 \mathrm{~b}$ shows an SHG autocorrelation trace of the compressed pulse after passing through the grating. When the separation of the grating-prism pair was $27 \mathrm{~cm}$, the pulse was compressed to the minimum width of $0.23 \mathrm{ps}$ ( $\mathrm{sech}^{2}$ shape) with weak side-lobes. It was then found that positive linear-chirp had occurred inside the cavity over the entire pulse envelope.

The compressed pulse serves as a probing pulse for the SHG cross-correlation measurement of the chirped pulse. Figure 8 shows the cross-correlation trace of the positively-chirped pulse at $P / P_{\text {th }}=1.5$. The correlation trace is best fitted by a 0.84 ps (FWHM) Gaussian shape (circles) in the leading side and by a 1.13 ps (FWHM) sech $^{2}$ shape in the trailing side (crosses). There exists a weak satellite peak at the trailing wing. Some asymmetry was always observed with a higher dye concentration regime, which also requires a higher pumping power.

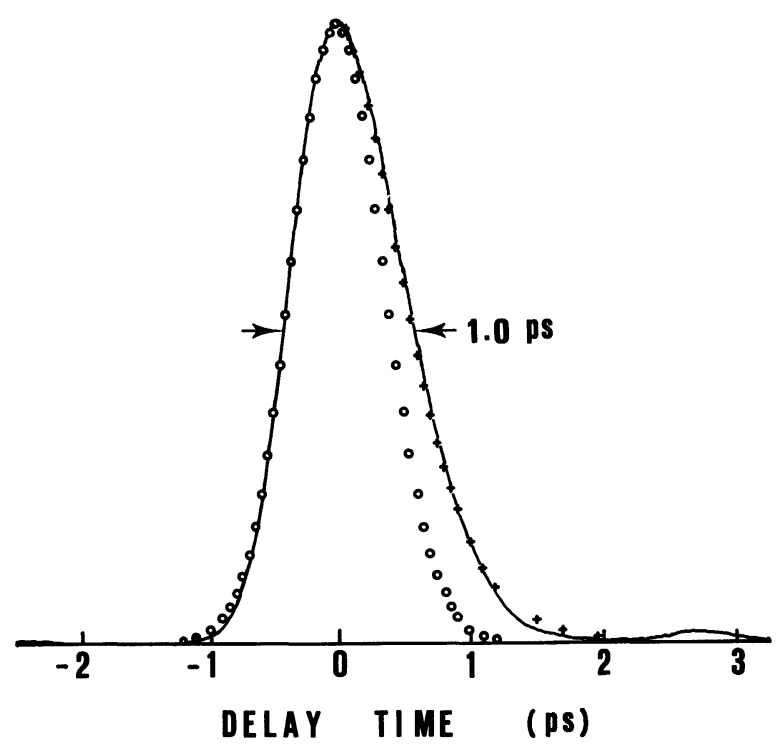

Fig. 8. - SHG cross-correlation trace of the positively chirped pulse corresponding to figure 7a. The correlation trace is best fitted by a 0.84 ps (FWHM) Gaussian shape (circles) in the leading side and by a 1.13 ps (FWHM) $\operatorname{sech}^{2}$ shape in the trailing side (crosses).

Figure 9 shows the wavelength dependence of the compressed pulse width at $P / P_{\mathrm{th}}=1.5$. This is very similar to that for the external fiber compression in figure 4 . The high peak power and the clean pulse shape are maintained in this wavelength region. 


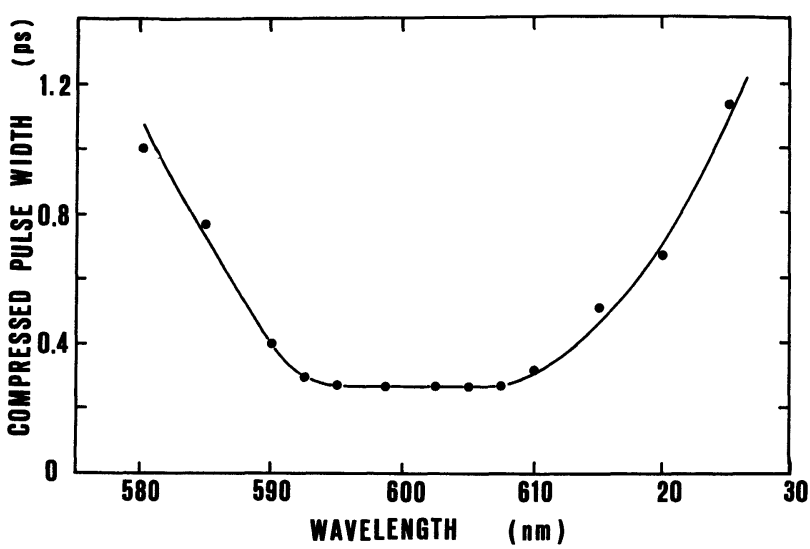

Fig. 9. - Wavelength dependence of the width of the compressed pulse corresponding to figure $7 \mathrm{~b}$.

3.3 INTRACAVITY SELF-PHASE MODULATION. Here, we discuss the origin of the positive linearchirp inside the cavity described in 3.2.

Recent studies show that intracavity self-phase modulation (ISPM) in cw mode-locked dye lasers plays an important role in the temporal and spectral behaviours of the output pulse, or in the pulseshaping [4, 9-19]. The mechanisms of ISPM reported are largely different depending on the laser system and the operating conditions. The evidence of the existence of slow SPM $\left(t_{\mathrm{p}} \ll \tau_{\mathrm{r}}\right)$ in the absorber dye solution has been shown with a hybridly modelocked dye laser, where the pulse was characterized by having both negative and positive chirps [9]. Here, $t_{\mathrm{p}}$ and $\tau_{\mathrm{r}}$ are the pulse width and the relaxation time of the nonlinear refractive index, respectively. It has also been reported that the behaviour of SPM in CPM ring dye lasers strongly depends on the absorber dye concentration (DODCI) [13]. In particular, under high concentrations of the absorber dye, which introduces shorter pulse width and higher pumping power, ultrafast SPM $\left(\tau_{\mathrm{r}} \ll t_{\mathrm{p}}\right)$ due to the electronic Kerr effect in the solvent rather than slow SPM originating from the dye solute becomes dominant.

The positive chirp observed, which is peculiar to our present laser system, can qualitatively be explained by this mechanism. The key point of our laser system is that a Bragg cell as a cavity dumper is introduced in the cavity as shown in figure 1 . The Bragg cell ( $3 \mathrm{~mm}$ thick) acts not only as a normal linear dispersive medium, but also as a nonlinear medium exhibiting fast SPM, because the optical field inside the cell is tightly focused as in the dye jet. Then, the Bragg cell will play a role similar to that of an optical fiber, considering a large round trip number inside the cavity.

First, to evaluate both the effects of slow SPM in the dye solution and fast SPM in the Bragg cell and in the dye solvent, we calculate the maximum frequency shift for both cases. For slow SPM, the maximum frequency shift $\Delta \omega_{\mathrm{s}}$ is given by

$$
\Delta \omega_{\mathrm{s}}=-\omega_{0} n_{2}^{\mathrm{s}} Z_{\mathrm{d}} A_{\mathrm{d}}^{2} / c \tau_{\mathrm{r}},
$$

where $n_{2}^{\mathrm{s}}$ and $Z_{\mathrm{d}}$ are the nonlinear refractive index of the dye solution and the thickness of the dye jet, respectively, and $A_{\mathrm{d}}$ and $\omega_{0}$ are the amplitude in the dye jet and the centre frequency of the light field, respectively.

For fast SPM, the maximum frequency shift $\Delta \omega_{\mathrm{f}}$ is calculated by assuming a Gaussian shape with the width $t_{\mathrm{p}}$ (FWHM) as

$$
\Delta \omega_{\mathrm{f}}=4(2 \ln 2)^{1 / 2} \mathrm{e}^{-1 / 2} \omega_{0} n_{2}^{\mathrm{f}}\left(Z_{\mathrm{b}} A_{\mathrm{b}}^{2}+Z_{\mathrm{d}} A_{\mathrm{d}}^{2}\right) / c t_{\mathrm{p}},
$$

where $n_{2}^{\mathrm{f}}$ and $Z_{\mathrm{b}}$ are the nonlinear refractive index and the thickness of the Bragg cell, respectively, and $A_{\mathrm{b}}$ is the field amplitude in the Bragg cell. The nonlinear refractive index of the dye solvent has been assumed to be the same as that of the Bragg cell. The ratio $R$ defined as $\Delta \omega_{\mathrm{f}} / \Delta \omega_{\mathrm{s}}$ is calculated from equations (1) and (2) as

$$
\begin{aligned}
R=4(2 \ln 2)^{1 / 2} \mathrm{e}^{-1 / 2}\left(\tau_{\mathrm{r}} / t_{\mathrm{p}}\right)\left(n_{2}^{\mathrm{f}} / n_{2}^{\mathrm{s}}\right) \times \\
\times\left[1+\left(Z_{\mathrm{b}} / Z_{\mathrm{d}}\right)\left(A_{\mathrm{b}} / A_{\mathrm{d}}\right)^{2}\right] .
\end{aligned}
$$

In equation (3), $\left(A_{\mathrm{b}} / A_{\mathrm{d}}\right)^{2}$ is equal to $\left(n_{\mathrm{d}} / n_{\mathrm{b}}\right)$ $\left(R_{\mathrm{d}} / R_{\mathrm{b}}\right)^{2}$, where $n$ 's and $R$ 's are the refractive indices and the radii of curvature of the mirror, respectively, and the suffices $d$ and $b$ denote the dye jet and the Bragg cell. With the following numerical values; $n_{2}^{\mathrm{f}}=1.1 \times 10^{-13}$ esu., $n_{2}^{\mathrm{s}}=2.5 \times 10^{-12}$ esu., $Z_{\mathrm{d}}=0.24 \mathrm{~mm}, Z_{\mathrm{b}}=3.6 \mathrm{~mm}, R_{\mathrm{d}}=50 \mathrm{~mm}$, $R_{\mathrm{b}}=100 \mathrm{~mm}, n_{\mathrm{d}} \simeq n_{\mathrm{b}}, \tau_{\mathrm{r}} \geqslant 10 \mathrm{ps}$, and $t_{\mathrm{p}}=1.0 \mathrm{ps}$, the value of $R$ is calculated from equation (3) to be 6.0. The magnitudes of $n_{2}^{\mathrm{s}}$ and $\tau_{\mathrm{r}}$ in the mixed dye solution are the same as reported previously [9]. Thus, the contribution of fast SPM to the chirp is expected to be dominant. Moreover, from the frequency independence of the compressed pulse width around the absorption peak $(600 \mathrm{~nm})$ of DQOCI shown in figure 9, the contribution of slow SPM showing the off-resonance dispersion may also be ignored. Further, we can calculate from equation (2) the maximum frequency shift per round trip due only to fast SPM by giving the beam spot size $S_{\mathrm{b}}$ in the Bragg cell and the internal peak power $I_{i}\left(=I_{0} / \eta\right)$, where $I_{0}$ and $\eta$ are the output peak power and the diffraction efficiency of the cavity dumper, respectively. With $I_{0}=1.8 \mathrm{~kW}, \eta=0.7$ and $S_{\mathrm{b}}=1.8 \times 10^{-6} \mathrm{~cm}^{2}, \Delta \omega_{\mathrm{f}} / \omega_{0}$ is calculated to be $4.0 \times 10^{-5}$. Assuming an effective round trip number of 100 , one obtains the total spectral broadening of $2.4 \mathrm{~nm}$ at $600 \mathrm{~nm}$, corresponding to the measured spectral width. The effective propagation length in 
all dispersive media with this round trip number amounts to $84 \mathrm{~cm}$.

It was also found that the repetition rate of the cavity dumper below which the output power approaches sufficiently to a steady state was $0.8 \mathrm{MHz}$. This corresponds also to an effective round trip number of 100 , which is the same order as that (210 times) discussed previously [9]. Therefore, if all the properties associated with output pulses reach a steady state over this round trip number in balance with gain, loss, linear and nonlinear dispersions, one can expect the spectral broadening over $5 \mathrm{~nm}$. Actually the laser spectral width $2.5 \mathrm{~nm}$ has been limited by the bandwidth limiting element of a single-plate birefringence filter.

Next, we consider a purely linear dispersion effect inside the cavity, which also causes the chirp and broadening of the pulse. For our input pulse of $0.23 \mathrm{ps}$ width and $600 \mathrm{~nm}$ centre wavelength, the output pulse width after propagating along a typical dispersive medium (quartz) of $84 \mathrm{~cm}$ long (effective length) is calculated to be $0.54 \mathrm{ps}$ from an existing formula [11]. Although this linear dispersion effect cannot be neglected in our laser system, the spectral behaviour and the pulse compression property in figure 9 depending on the peak intensity can no longer be explained from the linear effect alone. The generation of the clean positive linear-chirp in our case implies an effective contribution of the coupling between the linear dispersion and SPM inside the cavity as in the external fiber. This point largely differs from the previous results [9]. Further, the dispersion of the multicoated dielectric mirrors can be neglected because the laser spectral width $(<3 \mathrm{~nm})$ is sufficiently narrow compared to that of the mirrors.

From these considerations, we can conclude that under the present operating conditions the main origin of the positive linear-chirp is the ultrafast SPM in the electronic Kerr media. When a material with larger nonlinear coefficient and fast response time (such as $\beta$-carotene) is introduced in the cavity, larger amount of chirp can be produced at lower peak power level and the pulse can be internally or externally compressed to the femtosecond region. Presently, a preliminary experiment using several samples as Kerr media is in progress. This approach is also attractive to generate well-controlled, transform-limited and chirped ultrashort pulses for new nonlinear spectroscopic studies.

\section{Application to new transient nonlinear spectros- copy.}

Finally, we briefly mention some spectroscopic application of our present laser system with emphasis on the usefulness of chirped pulses for relaxation studies.
Since ultrafast relaxation processes in condensed matter are very complicated in general, it is necessary to develop theoretical and experimental studies from different approaches in time-domain and/or in frequency-domain spectroscopy. Recently, considerable advances in ultrashort pulse laser technology have made it possible to measure directly the ultrafast relaxation time in the time-domain.

Previously, we demonstrated the measurement of femtosecond relaxation times ( $\leq 100 \mathrm{fs}$ ) in organic dye solutions by transient nonlinear spectroscopy based on the four-wave mixing in k-space with two input pulses by using a passively mode-locked subpicosecond dye laser [20]. Such a subpulse-width resolution in the femtosecond region has been achieved with the measurement of peak-shift between the two correlation traces associated with two scattered light outputs. A similar study has been made by an extended scheme with three input pulses which improves the method [21].

In conventional transient spectroscopy using transform-limited ultrashort pulses, however, the timeresolution is eventually limited by the pulse width of light sources or the pulse broadening effect in the measurement system. To avoid this difficulty, a new method of transient nonlinear spectroscopy using non-transform-limited light has been proposed and developed by our group and others [2, 3, 22-31], where the time-resolution is governed by the correlation time $\tau_{c}$ instead of the pulse width of light sources. Incoherent and phase modulated pulses with broad bandwidth $(\Delta \omega)$ are such kind of light having $\tau_{c}\left(\sim \Delta \omega^{-1}\right)$ much shorter than the pulse width $t_{\mathrm{p}}$. While a number of experimental works demonstrating the ultrafast relaxation study with incoherent light have been reported, those with chirped pulses have not appeared yet. Our laser system producing both nearly transform-limited and linearly-chirped pulses of broadband and clean character is useful for an experimental demonstration using chirped pulses and for comparison of the results with those using transform-limited pulses.

The configuration of our present experimental

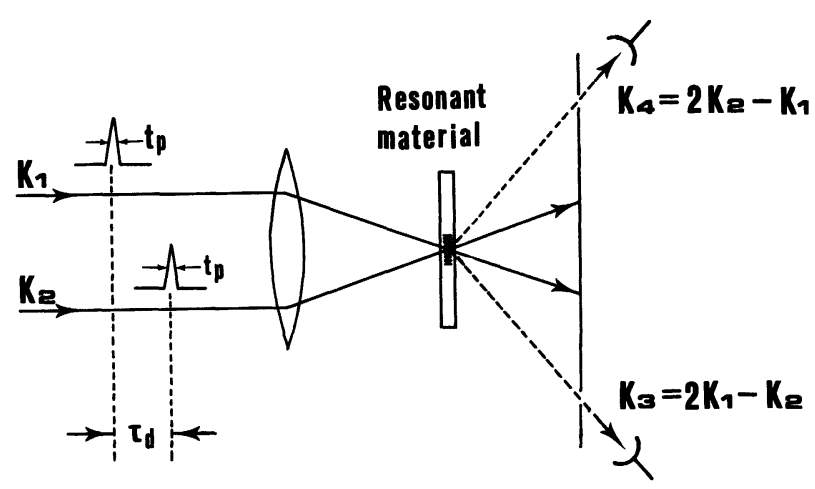

Fig. 10. - Configuration of the four-wave mixing with two incident beams for dephasing time measurements. 
study of transient nonlinear spectroscopy based on the degenerate four-wave mixing with two incident light beams is shown in figure 10 . When two light beams with wavevectors $\mathbf{k}_{1}$ and $\mathbf{k}_{2}$ are incident on a resonant material system, resulting population grating with wavevectors $\pm\left(\mathbf{k}_{1}-\mathbf{k}_{2}\right)$ scatters the incident light fields to produce two output beams in the new directions $k_{3}=2 \mathbf{k}_{1}-\mathbf{k}_{2}$ and $\mathbf{k}_{4}=2 \mathbf{k}_{2}-\mathbf{k}_{1}$. The correlation curve, i.e., the time-integrated intensity of one of the output beams as a function of the delay time $\tau_{\mathrm{d}}$ between two incident beams, gives information on dephasing time $T_{2}$, if the condition $T_{2} \geq t_{\mathrm{p}}$ or $\tau_{\mathrm{c}}$ holds. For the measurement of the peak shift of the correlation curve, simultaneous display of two correlation curves associated with the $\mathbf{k}_{3}$ and $\mathbf{k}_{4}$ beams is convenient.

The samples used as resonant materials are a dye solution of DODCI and a semiconductor $\left(\mathrm{CdS}_{x} \mathrm{Se}_{1-x}\right)$ doped glass filter (Corning 2-61). The laser wevelength can be tuned within the width at half-maximum of the absorption band $\left(S_{0} \rightarrow S_{1}\right)$ for the dye or around the band-edge for the glass filter. Two incident beams making an angle of $2^{\circ}$ are focused by a lens of $f=15 \mathrm{~cm}$ into a sample cell of $1 \mathrm{~mm}$ thick. All experiments were carried out at room temperature.

As a first experiment, nearly transform-limited incident pulses with parallel polarization were used for DODCI dye solution, and the result is shown in figure 11. Although exponential decay component arising from relaxation was not detected in the correlation curve, a clear peak shift between the two curves was measured to be $71 \mathrm{fs}$. To check the existence of thermal grating effect, orthogonal polarization scheme for the two incident beams was also tried. Measured correlation curves are shown in figure 12. Although the output signal was a factor of ten smaller than that for the parallel polarization scheme, the curves show a clearly resolved peak shift of $65 \mathrm{fs}$ in approximate agreement with that for the parallel polarization scheme. Thus, the peak shift is supposed to be insensitive to the thermal effect in this dye. Theoretical analysis has shown that this shift is a sensitive manifestation of relaxation effects in the resonant material $[20,21]$. In general, this shift depends on both $T_{1}$ (population relaxation time) and $T_{2}$, and its calculated results based on an inhomogeneously broadened two-level model have already been presented [20]. Existence of crossrelaxation and multi-level effects will further complicate this dependence. Nevertheless, we can make rough estimation of $T_{2}$ from the measured shift with the aid of the theory, because the shift comparable to the pulse width is dominantly governed by $T_{2}$. Thus, we can estimate the value of $T_{2}$ in this dye solution to be $20-50 \mathrm{fs}$.

Next, we examined the case of non-transformlimited light by using chirped pulses produced in an

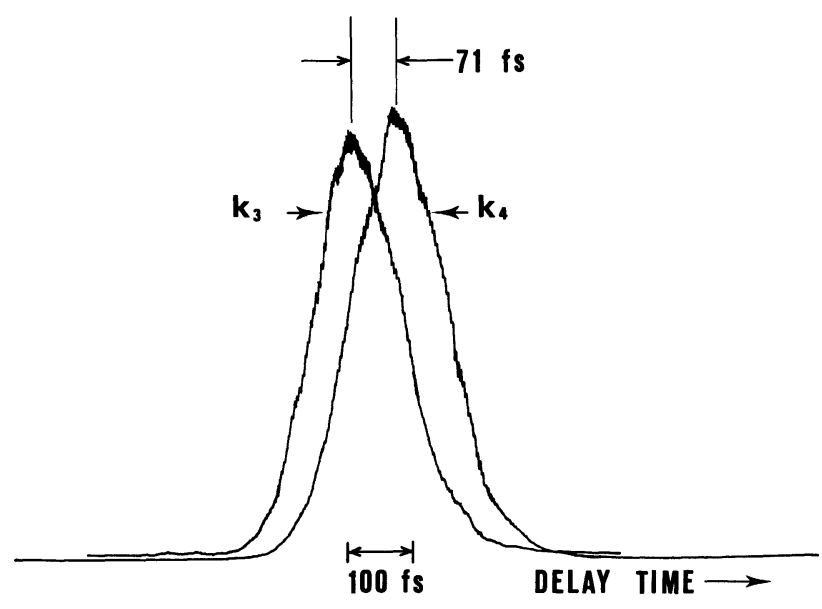

Fig. 11. - Measured correlation curves for two output beams with $\mathbf{k}_{3}$ and $\mathbf{k}_{4}$ of transient four-wave mixing with two incident transform-limited pulses having parallel polarization. The sample is DODCI dye in ethanol.

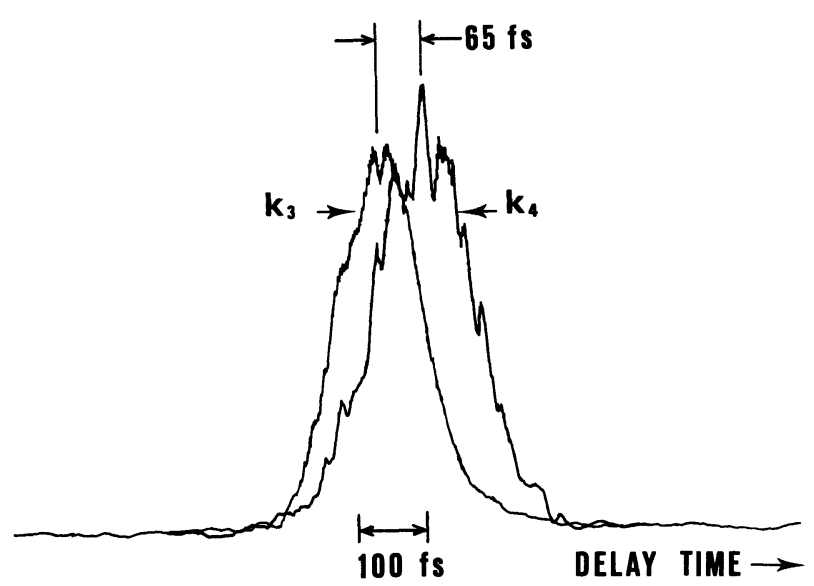

Fig. 12. - Measured correlation curves for two output beams with $\mathbf{k}_{3}$ and $\mathbf{k}_{4}$ of transient four-wave mixing with two incident transform-limited pulses having orthogonal polarization. The sample is DODCI dye in ethanol.

optical fiber. The correlation time corresponding to the measured spectral width $(5 \mathrm{~nm})$ becomes $110 \mathrm{fs}$, being much shorter than the pulse width $(8 \mathrm{ps})$. Even with this light source, correlation curves essentially similar to figure 11 were obtained as shown in figure 13, although the measured peak shift of 59 fs and the width of the correlation trace are somewhat narrower compared to the case of transform-limited pulse. In this experiment, polarizations of the two beams were set to be parallel. It has already been shown that a chirped pulse plays a similar role in this type of four-wave mixing to an incoherent light having the same correlation time and provides much higher time-resolution than the light duration $[2,3]$. The result of figure 13 gives experimental evidence for this prediction. A preliminary calculation of the peak shift in the correlation curve for chirped input 


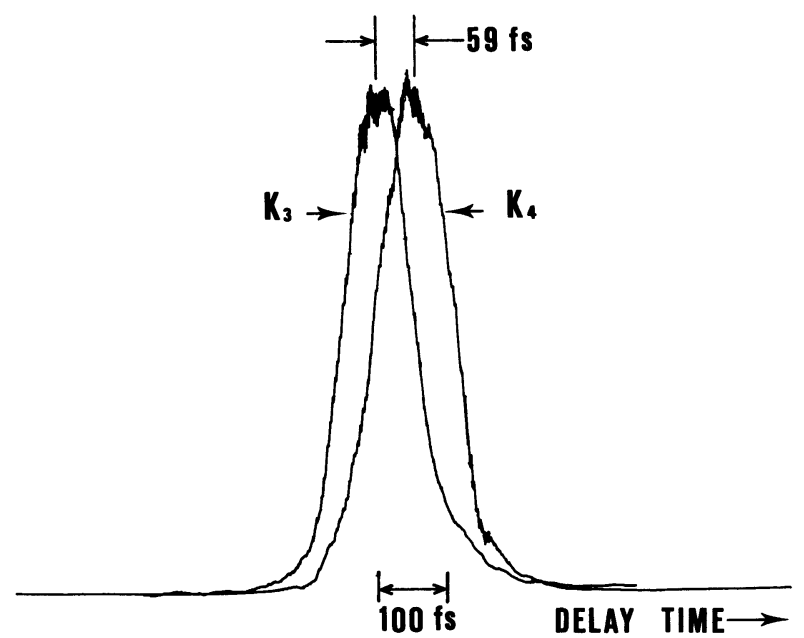

Fig. 13. - Measured correlation curves for two output beams with $\mathbf{k}_{3}$ and $\mathbf{k}_{4}$ of transient four-wave mixing with two incident linearly chirped pulses having parallel polarization and $8 \mathrm{ps}$ width. The sample is DODCI dye in ethanol.

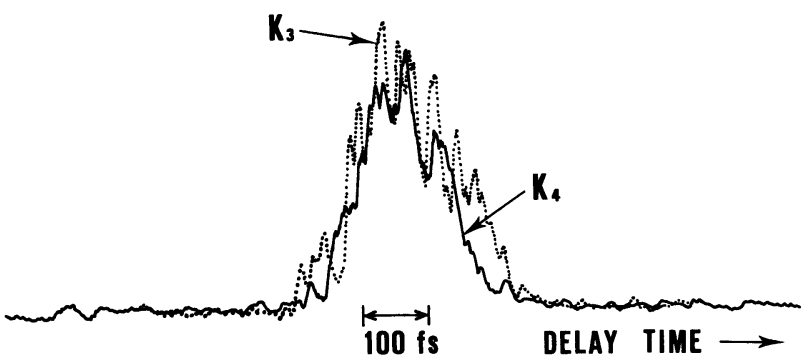

Fig. 14. - Measured correlation curves of transient fourwave mixing with two incident transform-limited pulses having parallel polarization. The sample is semiconductor doped glass filter $\mathrm{CdS}_{x} \mathrm{Se}_{1-x}$.

pulses shows essentially similar $T_{2}$ dependence to that for transform-limited pulses, although $T_{1}$ dependence and detailed behaviours are fairly different. The value of $T_{2}$ thus estimated is $30-40 \mathrm{fs}$, being consistent with that using transform-limited pulses. This result implies that the nonlinear spectroscopic method using chirped pulses is really effective for the relaxation study with high time-resolution. More improved studies of both theoretical and exper- imental are in progress, and the details will be given elsewhere.

In addition to the dye solution, we have also tried to measure the dephasing time of a semiconductor doped glass filter $1 \mathrm{~mm}$ thick. In this case, the output signal was about 20 times smaller than that for DODCI (Fig. 14). Due to the low signal-to-noise ratio the peak shift could no longer be detected within the time-resolution limit of about $20 \mathrm{fs}$, indicating very fast phase relaxation.

\section{Summary and conclusion.}

We have reported a well-controlled ultrashort pulse laser source suitable for studying a new method of nonlinear spectroscopy with chirped pulses as well as the conventional one with transform-limited pulses.

This laser system is based on a hybridly modelocked, cavity dumped, $\mathrm{cw}$ dye laser coupled with a single-stage fiber compressor. The pulse characteristics can effectively be controlled by the pumping power level and the absorber dye concentration to produce tunable, clean transform-limited or clean linearly-chirped pulses in a long lifetime operation. The positive linear-chirp inside the cavity, which occurs under certain conditions, has been attributed to ultrafast self-phase modulation in the Bragg cell and the dye solvent, being specific to our cavity configuration.

Our present laser system has also been applied to transient nonlinear spectroscopy to demonstrate that a chirped pulse behaves like a transform-limited pulse with the same spectral width in observing relaxation effects in resonant materials, and then provides high time-resolution.

Further improvements will make this type of laser system useful for spectroscopic applications on a shorter femtosecond time-scale over a broad frequency region.

\section{Acknowledgments.}

We would like to thank T. Tokizaki for his help in performing measurements and for discussions. This work was partially supported by a Grant-in-Aid for Scientific Research (light wave sensing) from the Ministry of Education, Science and Culture of Japan.

\section{References}

[1] See, for example, Ultrafast Phenomena $V$, Eds G. R. Fleming and A. E. Siegman (Springer-Verlag) 1986.

[2] Yajima, T., Morita, N. and Ishida, Y., J. Opt. Soc. Am. B 1 (1984) 526.
[3] Yajima, T. and Morita, N., in Methods of Laser Spectroscopy, Eds Y. Prior, A. Ben-Reuven and M. Rosenbluh (Plenum Publishing Corporation) 1986, p. 75.

[4] Valdmanis, J. A., Fork, R. L. and Gordon, J. P., Opt. Lett. 10 (1985) 131. 
[5] Ishida, Y. and Yajima, T., Opt. Commun. 58 (1986) 355.

[6] Nakatsuka, H., GrischKowsky, D. and BALANT, A. C., Phys. Rev. Lett. 47 (1981) 910.

[7] Grischkowsky, D. and Balant, A. C., Appl. Phys. Lett. 41 (1982) 1.

[8] Tomlinson, W. J., Stolen, R. H. and Shank, C. V., J. Opt. Soc. Am. B 1 (1984) 139.

[9] Ishida, Y., Naganuma, K. and YaJima, T., IEEE J. Quantum Electron. QE-21 (1985) 69.

[10] Watanabe, A., Saito, H., Ishida, Y. and YaJima, T., Opt. Commun. 63 (1987) 320.

[11] Dietel, W., Döpel, E., KüHlke, D. and Wilhelmi, B., Opt. Commun. 43 (1982) 433.

[12] Fontaine, J. J., Dietel, W. and Diels, J.-C., IEEE J. Quantum Electron. QE-19 (1983) 1467.

[13] IshidA, Y., Naganuma, K., YaJima, T. and Lin, L. H., in Ultrafast Phenomena $I V$, Eds D. H. Auston and K. B. Eisenthal (Springer-Verlag) 1984 , p. 69.

[14] Rudolph, W. and Wilhelmi, B., Opt. Commun. 49 (1984) 371.

[15] Martinez, O. E., Fork, R. L. and Gordon, J. P., J. Opt. Soc. Am. B 2 (1985) 753.

[16] Diels, J.-C., Dietel, W., Fontaine, J. J., Rudolph, W. W. and Wilhelmi, B., J. Opt. Soc. Am. B 2 (1985) 680.

[17] Dietel, W., Fontaine, J. J. and Diels, J.-C., Opt. Lett. 8 (1983) 4.
[18] Heppner, J. and Kuhl, J., Appl. Phys. Lett. 47 (1985) 453.

[19] Dawson, M. D., Boggess, T. F., Garvey, D. W. and Smirl, A. L., Opt. Commun. 60 (1986) 79.

[20] Yajima, T., IshidA, Y. and TaIRA, Y., in Picosecond Phenomena II, R. M. Hochstrasser, Eds W. Kaiser and C. V. Shank (Springer-Verlag) 1980 , p. 190.

[21] Weiner, A. M., De Silvestri, S. and Ippen, E. P., J. Opt. Soc. Am. B 2 (1985) 654.

[22] Asaka, S., Nakatsuka, H., Fujiwara, M. and Matsuoka, M., Phys. Rev. A 29 (1984) 2286.

[23] Morita, N. and Yajima, T., Phys. Rev. A 30 (1984) 2525.

[24] Beach, R. and Hartmann, S. R., Phys. Rev. Lett. 53 (1984) 663.

[25] Fujiwara, M., Kuroda, R. and Nakatsuka, H., J. Opt. Soc. Am. B 2 (1985) 1634.

[26] Beach, R., De Beer, D. and Hartmann, S. R., Phys. Rev. A 32 (1985) 3467.

[27] Defour, M., Keller, J. C. and Le Gouët, J. L., J. Opt. Soc. Am. B 3 (1986) 544.

[28] Golub, J. E. and Mossberg, T. W., J. Opt. Soc. Am. B 3 (1986) 554.

[29] Tomita, M. and Matsuoka, M., J. Opt. Soc. Am. B 3 (1986) 560.

[30] Morita, N., Torizuka, K. and Yajima, T., J. Opt. Soc. Am. B 3 (1986) 548.

[31] Hattori, T., Terasaki, A. and Kobayashi, T., Phys. Rev. A 35 (1987) 715. 